\title{
Adaptive friction compensations for mechanical systems with measurement delay
}

\author{
Caner Odabaş ${ }^{1,2}$ (1) and Ömer Morgül'
}

\begin{abstract}
Application performance of mechanical positioning systems might not coincide with the theory, mainly due to nonlinearities or imperfections of system models. Although it is sometimes possible to ignore these mismatches, systems generally suffer from performance degradation or even instability eventually. Especially, friction force and time delay are two major factors of these undesired effects. Hence, in this paper, Smith predictor-based controllers and an adaptive Coulomb friction observer are designed to enhance position tracking performance of a mechanical system including time delay. In fact, implemented hierarchical control scheme provides two-degree of freedom to control both velocity and position separately. The proposed observer structure is mainly motivated by the Friedland-Park observer but could be considered as an extension of it which characterizes a general class of nonlinear functions for friction estimation. To assure its functionality with delayed measurements, different velocity predictor schemes are designed and their performances are compared. As a guideline for observer design, some conditions for exponential stability and robustness analysis are presented. Simulation results demonstrate that the proposed control system enhances the tracking performance even when the actual friction is a compound of various static and dynamic terms.
\end{abstract}

\section{Keywords}

Friction observer, time delay, Smith predictor, controller parametrization, hierarchical position control, adaptive control

\section{Introduction}

Friction is a natural phenomenon that can directly effect system performance and stability. Therefore, in controller design process, it may be necessary to employ convenient cancellation techniques for a good position tracking. To this end, there are lots of different approaches in friction compensation; see, for example, Armstrong-Hélouvry et al. (1994) and the references therein. Although friction modeling and identification are vast areas, simple Coulomb model describes the most basic and dominant component of friction at the steady state. Besides, it is possible to make this static model be more realistic by adding Stribeck effect and viscous coefficient (Olsson et al., 1998). However, these static models are limited to describe friction since it is highly nonlinear. On the other hand, dynamic models can exhibit stick-slip motion, breakaway force, presliding behavior, and so forth, in a better extend. For instance, Dahl (1968) developed a simulation model for rolling and sliding behavior assuming that the origin of friction is in quasi static contact bonds that are continuously formed and subsequently broken. In this model, the friction force is only a function of the displacement and the sign of velocity. This important property associated with the model, so called rate independence, provides an advantage to model hysteresis and pre-sliding displacement. Nevertheless, it does not involve viscous friction, stiction and Stribeck effect. Afterwards, Bliman and Sorine (1993) utilized linear space invariant differential operators in order to obtain a dynamical friction model. By this, their motivation was to extend Dahl model to capture stiction and Stribeck effect. To this end, their model managed to connect a fast and slow Dahl model in parallel. Unfortunately, although this model compromises stick friction, it only provides an insufficient transient Stribeck effect after a change of the direction of motion that is not present in the steady state. Another generalization of Dahl model is the widely used LuGre friction model. Canudas de Wit et al. (1995) defined an internal state variable $z_{d}$ to determine the average deflection of the asperities and includes rate dependent friction phenomena such as varying break-away force and frictional lag. Compared with Dahl and Bliman-Sorine models, LuGre model achieves the inclusion of viscous friction and Stribeck effect at the same time.

After an appropriate model describing the friction well enough is obtained, an equivalent opposing force having same parameters can be superposed to control input to cancel out

\footnotetext{
'Department of Electrical and Electronics Engineering, Bilkent University, Turkey

${ }^{2}$ Radar, Electronic Warfare and Intelligence Systems Department, ASELSAN Inc., Turkey

\section{Corresponding author:}

Caner Odabaaş, Department of Electrical and Electronics Engineering, Bilkent University, Bilkent, Ankara, TR-06800, Turkey.

Email: canero@ee.bilkent.edu.tr
} 
the friction nonlinearities (Lischinsky et al., 1999). However, precise dynamical parameter identification can be challenging. Besides, these parameters can vary according to the material properties, environment, temperature, position, velocity and time that is why observer based adaptive techniques for friction compensation is very popular in the literature. For instance, Lee and Tomizuka (1996) used a friction compensator firstly for static coefficients in either feedback or feedforward loop. Then, the difference between actual output and output of the nominal model was regarded as an equivalent disturbance applying to the system model, which is why a Q-filter based disturbance observer was attached to improve friction cancellation performance. Xie (2007) developed a sliding-mode observer to estimate the internal friction state of LuGre model. Based on estimation, an adaptive controller drives the position and velocity of motor to track the reference signals. Ray et al. (2001) utilized extended Kalman-Bucy filter to estimate friction. Actually, this technique can work well in the absence of any friction model, therefore it can be treated as a non-model-based cancellation approach. For online estimation of parameters of not only friction but also other nonlinear uncertainties, Chen et al. (2020) and Chen et al. (2013) used projection typed adaptation laws with least square based algorithms. Lastly, an adaptive observer, namely FriedlandPark observer, was utilized to estimate Coulomb parameter (Friedland and Park, 1992; Tafazoli et al., 1998). It seems that this observer improves the performance even when the actual friction does not conform to Coulomb model only.

We note that although our proposed observer structure is motivated by the work of Friedland-Park, there are considerable differences as well. First, the nonlinear observer structure proposed here is quite general and contains the one proposed in Friedland and Park (1992) and Friedland and Mentzelopoulou (1992) as a special case. Indeed, we characterize a general class of nonlinearities that achieve friction estimation under certain conditions (see Remark 1). We also provide several stability results related to the proposed observer structure, which shows the exponential stability of the friction estimator under certain conditions (see Lemma 1 and Lemma 2). Also, note that the time delay is not considered in Friedland and Park (1992) and Friedland and Mentzelopoulou (1992), whereas here we consider the effect of measurement delays and their effect on friction cancellation. Furthermore, we provide a result that shows that our proposed scheme is sufficiently robust against the measurement errors caused by the delay (see Lemma 3 and Remark 4). Similar remarks could also be stated between our first work (Odabaş, 2014) and the present one. First of all, Odabaş (2014) utilizes mainly the observer proposed by Friedland and Park (1992). Moreover, Odabaş (2014) does not contain a detailed stability analysis as compared with the work here. Finally, although a time delayed system has been considered previously, the actual velocity measurements required for friction cancellation are assumed to be available. This is not always realistic and to overcome this issue, here, we propose a procedure to estimate the actual velocities based on delayed measurements. Correspondingly, the possible effects of velocity measurement errors are considered and a result related to the robustness of proposed scheme is provided. Hence, in this sense, the differences between Friedland and Park (1992) and our present work mentioned above also apply to our past work (Odabaş, 2014) as well.

Time delay is another issue affecting performance and stability in mechanical systems. Time delay, also known as dead time, may emerge due to signal transmission, communication, processing, analysis or measurement. In this case, it is hard to design a controller since time delay introduces infinitely many poles into closed loop linear systems. Therefore, Smith (1957) proposed a special predictor to ease controller design process. In this case, it is possible to design a controller as if the plant is delay free. However, Watanabe and Ito (1981) proved that constant disturbance rejection cannot be achieved by using original Smith predictor structure. Afterwards, many other modifications have been made to improve the performance of Smith predictor in various application fields. For instance, Astrom et al. (1994) introduced a new form for the Smith predictor aiming to decouple disturbance and set-point response from each other. In Majhi and Atherton (2000), an auto tuning algorithm is developed to estimate Smith predictor parameters; however, it requires no modeling error in the assumed plant transfer function. In reality, the perfect representation of the plant might not be always available; therefore, Matausek and Micic (1996) have made an addition of extra feedback path from the difference of plant output and the model output to the control input aiming at higher order process control. Later, in Matausek and Micic (1999), it is suggested to replace proportional controller in predictor structure with a lead/lag controller in order to have fast disturbance rejection. Furthermore, Cicco et al. (2011) studied a congestion control problem such that there is a bounded uncertainty in dead time. For this problem, they utilized a geometric approach to design a Smith predictor plus proportional controller for the network system. For the control of a robot arm, Taşdelen and Özbay (2013) employ internal model principle for Smith predictor-based velocity and position controller design. Internal model control (IMC) principle implies that to reject impacts of disturbances and to track a reference signal, the controller structure should include the copies of disturbance and reference signal generators (Francis and Wonham 1976). Hence, Taşdelen and Özbay (2013) proposed a hierarchical position control system to achieve desired set point tracking and robustness criterion based on IMC principle.

As stated in Olsson and Astrom (1996), an effective friction compensation scheme generally requires velocity measurements with a good resolution and small time delay. Mostly, measurement delay is introduced by sampling, data collection system/sensor design (i.e. encoder resolution, complex communication protocols etc.) and velocity estimation if direct measurement of it is not possible. Apart from other studies, in this paper friction and measurement delay in a feedback control system are considered simultaneously. In the subsequent sections of this paper, we first revisit friction observer design for delay free systems. We show that it is necessary to make a velocity prediction to estimate the friction accurately since measurement delay deteriorates the performance of the observer and limits its bandwidth significantly. To this end, we propose different prediction approaches. Then, Smith predictor-based controllers are designed using controller parametrization and pole placement methods in 


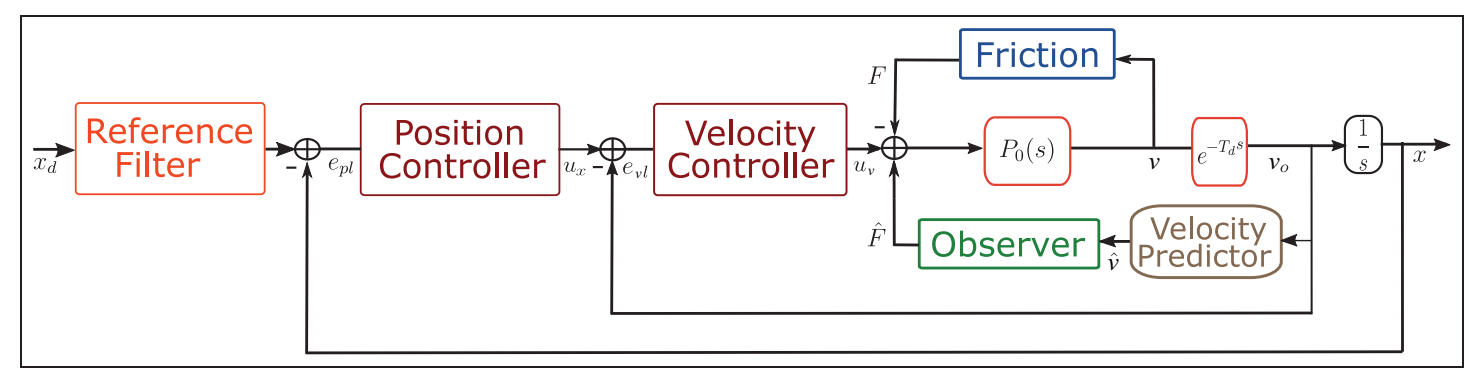

Figure I. Hierarchical closed loop position control system.

order to control position and velocity loops separately according to performance and robustness requirements. Therefore, proposed hierarchical closed loop position control strategy provides two degree of freedom as in Figure 1. Finally, a reference filter is designed to smooth the transition response and increase the position tracking performance at the steady state. Simulation results demonstrate that attached observer is capable of compensation of not only Coulomb but also other friction terms as well in the presence of time delay.

To summarize, the main contributions of our work are as follows. First, we generalize the observer structure of Friedland-Park and characterize a general class of nonlinear functions that could be utilized in this structure. Then, various stability results which show that the proposed structure is exponentially stable under certain cases are provided. Furthermore, the effect of measurement delay on friction compensation is considered and various schemes are proposed to estimate the actual velocity based on delayed velocity measurements. Lastly, the effect of velocity measurement errors on friction compensation is analysed. We show that our proposed scheme has certain robustness against such errors.

\section{Adaptive friction observer design Observer design for delay free systems}

In a simple mechanical system, applied force is control input whereas position and/or velocity is the output. Assuming that the system is delay free, equations of motion under presence of friction can be written as follows

$$
\begin{aligned}
\dot{x} & =v, \\
M \dot{v} & =-F\left(v, a_{c}\right)+\hat{F}\left(v, \hat{a}_{c}\right)+u .
\end{aligned}
$$

where, $M, u, x$ and $v$ stand for total mass, control input, position and velocity respectively. There are lots of different approaches to model the friction force in the literature; however, Coulomb coefficient, $a_{c}$, is a common and fundamental term in each of them. Thus, observer depicted in Figure 2 mainly tries to estimate and cancel Coulomb friction. Consequently, existing and estimated friction can be formulated as $F\left(v, a_{c}\right)=M a_{c} \operatorname{sgn}(v)$ and $\hat{F}\left(v, \hat{a}_{c}\right)=M \hat{a}_{c} \operatorname{sgn}(v)$, respectively.

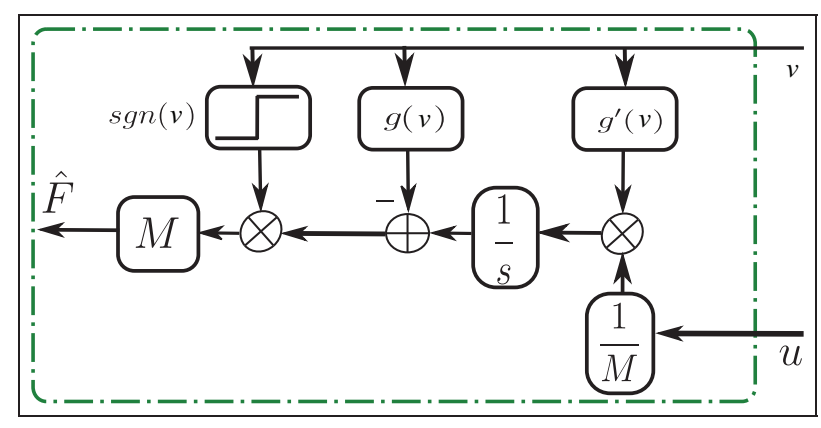

Figure 2. Structure of a generalized Coulomb observer.

To design the observer, let $g(v): \mathbf{R} \mapsto \mathbf{R}$ be an appropriate differentiable function yet to be determined. Inspired by Friedland and Park (1992), we propose the following

$$
\begin{aligned}
& \dot{z}=g^{\prime}(v) \frac{u}{M}, \\
& \hat{a}_{c}=z-g(v) .
\end{aligned}
$$

Here, $z$ is the internal state of the observer and $\hat{a}_{c}$ is the estimation of Coulomb friction coefficient. To analyze the performance of the observer given by (3), (4), let us define the estimation error as:

$$
e=a_{c}-\hat{a}_{c}
$$

Then, assuming that $a_{c}$ is constant, error dynamics can be derived as follows

$$
\begin{aligned}
\dot{e} & =-\dot{\hat{a}}_{c} \\
& =-\dot{z}+g^{\prime}(v) \dot{v} \\
& =-\frac{g^{\prime}(v) u}{M}+\frac{g^{\prime}(v)\left[\hat{F}\left(v, \hat{a}_{c}\right)-F\left(v, a_{c}\right)+u\right]}{M} \\
& =-\frac{g^{\prime}(v)}{M}\left[F\left(v, a_{c}\right)-\hat{F}\left(v, \hat{a}_{c}\right)\right] \\
& =-g^{\prime}(v) \operatorname{sgn}(v) e .
\end{aligned}
$$

Remark 1: If $g^{\prime}(v) \operatorname{sgn}(v) \geqslant 0 \forall v \neq 0$, then under some conditions one can show the asymptotic stability of the error dynamics given by (6). Quite a large class of functions $g(\cdot)$ satisfy these conditions. To characterize these, let us define 
the following class of functions. Consider a function $h: \mathbf{R} \mapsto \mathbf{R}$ that satisfies the following condition

$$
\alpha v^{2} \leqslant v h(v) \leqslant \beta v^{2} \forall v
$$

where $\beta \geqslant \alpha$. Such functions have a graph in Cartesian axes whose boundaries are given by the lines $y=\alpha v$ and $y=\beta v$ and we say in such a case that $h(\cdot)$ belongs to a sector $[\alpha, \beta]$, or simply $h \in[\alpha, \beta]$; see Khalil (2002) for more details. Note that if $\alpha=0, \beta=\infty$, then the functions $h \in[0, \infty]$ have a graph that lies entirely in first and third quadrant of Cartesian axes. Since $\operatorname{sgn}(\cdot) \in[0, \infty]$, it is clear that for any function $g$ such that $g^{\prime}(\cdot) \in[0, \infty]$, we will have $g^{\prime}(v) \operatorname{sgn}(v) \geqslant 0$ $\forall v$. Clearly, a large class of functions $g(\cdot)$ satisfy this condition. For example, if we choose $g(v)=\frac{1}{2} k v^{2}$, then $g^{\prime}(v)=k v$, which belongs to sector $[0, \infty]$ when $k>0$. Indeed, in this case we have $g^{\prime}(v) \operatorname{sgn}(v)=k|v| \geqslant 0$. Another such a function is $g(v)=k \ln (\cosh (\alpha v))$ where $k>0$ and $\alpha>0$ are arbitrary constants. In this case, $g^{\prime}(v)=k \alpha \tanh (\alpha v) \in[0, \infty]$. For the choice of $g(v)=k|v|, k>0$, we have $g^{\prime}(v) \operatorname{sgn}(v)=k$ and in this case the error equation given by (6) has the solution $e(t)=e^{-k t} e(0)$, provided that $v \neq 0$. In Friedland and Park (1992), the function $g(v)=k|v|^{\mu}$ is utilized, which yields $g^{\prime}(v)=k \mu|v|^{\mu-1} \operatorname{sgn}(v) \in[0, \infty]$ for any positive constants $k$ and $\mu$.

Note that although for particular choice of $g(v)=k|v|^{\mu}$, the well-known observer given in Friedland and Park (1992) will be attained; as a novel contribution, we also show that it is possible to use other alternatives of $g(v)$ as far as they satisfy Lemma 1.

Under certain mild assumptions, one can show the stability of the error dynamics given by (6) by using the standard Lyapunov stability analysis.

Lemma 1: Consider the system given by (1)-(6). Assume that $g^{\prime}(v) \in[0, \infty]$ is a nonlinear function with $g(v) \neq 0$ and $g^{\prime}(v) \neq 0$ for $v \neq 0$. Under these constraints, if $|v| \geqslant \alpha \forall t$, then the error dynamics given by (6) is exponentially stable, that is, $e(t) \rightarrow 0$ with an exponential decay

Proof: Note that $g^{\prime}(v) \operatorname{sgn}(v) \geqslant 0$ and with the given assumptions it easily follows that if $|v| \geqslant \alpha>0$ then, $g^{\prime}(v) \operatorname{sgn}(v) \geqslant \beta$ for some $\beta>0$. Let us define the following Lyapunov function

$$
V(e)=\frac{1}{2} e^{2}
$$

By differentiating (8) and using (6) we obtain

$$
\dot{V}=e \dot{e} \leqslant-\beta e^{2}=-2 \beta V
$$

Hence, $V(t) \leqslant e^{-2 \beta t} V(0)$ and therefore we have

$$
|e(t)| \leqslant e^{-\beta t}|e(0)|
$$

Remark 2: Although the applicability of Lemma 1 seems to be limited since it requires that $|v| \leqslant \alpha>0$; nevertheless, it could be utilized in various meaningful applications such as unit step tracking in velocity loop, ramp tracking in position loop. Note that in these cases, it is expected that condition $|v| \geqslant \alpha$ hold sufficiently long period of time. Indeed, if we choose $g(v)=k v^{2}$, then we have $g^{\prime}(v) \operatorname{sgn}(v)=2 k|v|$, hence for $|v|>\alpha, g^{\prime}(v) \operatorname{sgn}(v) \geqslant \beta$ is satisfied with $\beta=2 k \alpha$.

A somewhat less restrictive assumption on $v(t)$, which could be related to persistency of excitation (Sastry and Bodson, 2011), could be given as below.

Lemma 2: Let $v(t)$ be the solution of (1)-(6) and let us define

$$
G(t)=g^{\prime}(v(t)) \operatorname{sgn}(v(t))
$$

Assume that there exist some $\alpha>0$ and $T>0$ such that the following holds

$$
\int_{t}^{t+T} \mathrm{G}(\mathrm{s}) \mathrm{d} s \geqslant \alpha, \forall t \geqslant 0
$$

Then, for the error dynamics given by (6), $e(t) \rightarrow 0$ as $t \rightarrow \infty$; moreover, the decay is exponential.

Proof: Note that the solution of (6) is given as

$$
e(t)=e^{-\int_{0}^{t} \mathrm{G}(\mathrm{s}) \mathrm{d} s} e(0) .
$$

Since the exponential term is always positive, (13) can be rewritten as

$$
|e(t)|=e^{-\int_{0}^{t} \mathrm{G}(\mathrm{s}) \mathrm{d} s}|e(0)| .
$$

Let $t=n T+\tau$ for some integer $n \in Z^{+}$and $0<\tau<T$. Then

$$
\int_{0}^{t} \mathrm{G}(\mathrm{s}) \mathrm{d} s=\int_{0}^{n T} \mathrm{G}(\mathrm{s}) \mathrm{d} s+\int_{n T}^{n T+\tau} \mathrm{G}(\mathrm{s}) \mathrm{d} s \geqslant n \alpha .
$$

where the fact that $G(s) \geqslant 0$ is used. Inserting (15) into (14) leads to

$$
|e(t)| \leqslant e^{-n \alpha}|e(0)| .
$$

As $t \rightarrow \infty, n \rightarrow \infty$ as well; hence, $|e(t)| \rightarrow 0$. As it can be seen from (16), the decay is exponential. In fact, since $n=\frac{t-\tau}{T}$ and $\tau<T$

$$
|e(t)| \leqslant e^{\alpha} e^{-\frac{\alpha t}{T}}|e(0)|
$$

Remark 3: Note that when $|v(t)| \geqslant \alpha>0$, the condition given by (11) also holds. On the other hand, when the signals are in the sinusoidal form, the condition $|v(t)| \geqslant \alpha$ may not hold whereas (11) may hold. Hence, Lemma 2 could be utilized in the sinusoidal signal tracking and/or rejection. Indeed, if we choose $g(v)=k v^{2}$, then $G(t)$ given by (11) satisfies $G(t)=2 k|v|$. If $v(t)=\sin (\omega t)$, then (12) is satisfied with $T=\pi / \omega$ and $\alpha=2 k / \omega$.

\section{Observer design for systems with time delay}

Previously, Odabaş and Morgül (2014) modified FriedlandPark observer assuming that time delay exists in plant itself not in measurement process and friction parameters are 
constant or slowly time varying. Therefore, observer includes a copy of the time delay attached to control input port, $u$ to make a fair computation using theoretical velocity derived from $u$ and actual velocity $v$. By this, observer can estimate friction by (3)-(4) since both estimated and existing friction forces are functions of actual velocity. Under these assumptions, friction cancellation is achieved for relatively small dead time. However, observer performance is limited since measurement delay and dynamical friction parameters are not considered sufficiently.

Specifically, in this paper, we focus on measurement/output delay instead of actuator/plant delay and provide some stability proofs for this case. In other words, compared with Odabaş and Morgül (2014), in this paper, delay in the closed loop feedback system is modeled in a different way; therefore, the observer in this case does not contain a copy of time delay.

When the velocity measurements are delayed, to compute the friction force accurately the actual velocity should be estimated. Indeed, as it can be seen in Figure 1, while the actual velocity $v(t)$ is used to generate the actual friction force, the friction estimator only utilizes the delayed velocity measurement $v_{o}(t)$. Utilizing this delayed measurement without a velocity prediction may result in performance degradation, and even in instability. Referring to Figure 1, the relation between the measured velocity $v_{o}(t)$ and the actual velocity $v(t)$ becomes

$$
v_{o}(t)=v\left(t-T_{d}\right) .
$$

Replacing $t$ with $t+T_{d}$, we see that obviously we need to have $v_{o}\left(t+T_{d}\right)$ to estimate the friction force accurately. Hence, the actual velocity $v(t)$ to calculate the friction force and observed velocity $v_{o}(t)$ are related as follows

$$
v(t)=v_{o}\left(t+T_{d}\right)
$$

Since we observe $v_{o}(t)$, but $v(t)$ is required to calculate the friction force, we need to estimate $v(t)$. Let us call $\hat{v}(t)$ as an estimation of $v(t)$, and define the velocity estimation error $e_{v}$ as

$$
e_{v}=v(t)-\hat{v}(t)
$$

Note that it is not necessary to make such a velocity prediction for Odabaş and Morgül (2014) since actuator/input delay appears in closed loop feedback system rather than measurement/output delay. In other words, while in Odabaş and Morgül (2014) $v(t)$ is assumed to be available, here we assume that only $v_{0}(t)$ is available from measurements.

As a first alternative to predict $v_{o}\left(t+T_{d}\right)$, one can simply utilize the definition of derivative as given below

$$
\dot{v}_{o}(t)=\lim _{T_{d} \rightarrow 0} \frac{v_{o}\left(t+T_{d}\right)-v_{o}(t)}{T_{d}} .
$$

Note that $\dot{v}_{o}(t)$ can be easily found since past velocity measurements have already been available. Then, $\hat{v}(t)$ can be estimated by using current value of $v_{o}(t)$ and its derivative $\dot{v}_{o}(t)$.
By using this approach, which is well known as Euler approximation, we can approximate $\hat{v}(t)$ as follows

$$
v(t)=v_{o}\left(t+T_{d}\right) \approx v_{o}(t)+T_{d} \dot{v}_{o}(t) .
$$

By considering $\hat{v}(t)$ is the estimation of $v(t)=v_{o}\left(t+T_{d}\right)$, we may utilize the following

$$
\hat{v}(t)=v_{o}(t)+T_{d} \dot{v}_{o}(t) .
$$

Now from (21) it follows that the predicted velocity error $e_{v}$ will be small if the time delay $T_{d}$ is sufficiently small.

Note that Euler method is a first order approximation and there are some higher order methods such as Runge-Kutta, midpoint and so forth (Butcher and Goodwin, 2008). For instance, Heun Method, a particular choice of Runge-Kutta Method, is a second order technique that uses Euler approximation at the initial step, that is

$$
\begin{aligned}
& v_{o}^{i}\left(t+T_{d}\right)=v_{o}(t)+T_{d} \dot{v}_{o}(t) \\
& v_{o}\left(t+T_{d}\right)=v_{o}(t)+\frac{T_{d}}{2}\left(\dot{v}_{o}(t)+\dot{v}_{o}^{i}\left(t+T_{d}\right)\right) .
\end{aligned}
$$

However, numerical derivative operation adds both positive and negative errors at the end of each step; therefore, it tends to be unstable. Although higher order approximations may provide better accuracy in calculation of derivative numerically, they become more sensitive to input uncertainties. In contrast to numerical integration, differentiation is an illconditioned process. Conditioning or condition number is a definition to measure amplification of the input perturbation at the output. Since high order numerical derivatives tends to provide larger condition numbers, they become vulnerable to input uncertainties as well (Chapra et al., 2010). Hence, in practice, filters are utilized to prevent large oscillations at the input of derivative action (Visioli, 2006). An appropriate filtering process and filter design are other wide research topics; however, a simple low pass filtered differentiator, $L(s)$ is designed for the scope of this paper as in Tafazoli et al (1998). While they employed this filter as a velocity prediction from position measurements, here in this study, we employed it to predict velocity based on delayed velocity measurements. Certainly, it is also possible to use such a filter for a velocity prediction from delayed position measurements in the absence of direct velocity output. Thus, we may use the following high-pass filter as a differentiator

$$
L(s)=\frac{s N}{s+N}
$$

where $N$ is high-pass filter coefficient. As $N$ increases, filter behavior converges to ideal derivative operation. Nevertheless, in this case, filter gain becomes larger for high frequency signals and generates larger variations. Hence, choice of $N$ should be done carefully realizing this trade-off Note that MATLAB also utilizes the same filtering to generate PID controllers. 
Alternatively, irrational $e^{-T_{d} s}$ can be approximated by a rational function using Pade approximation such that

$$
e^{-T_{d} s} \approx R(s)=\frac{\sum_{k=0}^{n}(-1)^{k} c_{k} T_{d}^{k} s^{k}}{\sum_{k=0}^{n} c_{k} T_{d}^{k} s^{k}}
$$

where $n$ is the order of approximation and coefficients are

$$
c_{k}=\frac{(2 n-k) ! n !}{2 n ! k !(n-k) !}, k=0,1, \ldots, n .
$$

Typically, as dead time increases, $n$ should be increased as well to keep approximation error low. On the other hand, for very large values of $n$, the relative magnitude $c_{0} / c_{n}$ of the coefficients in (27) become very large; therefore, some numerical analysis/simulation difficulties can arise (Ozbay, 1999). Furthermore, a high order approximation becomes to produce clustered poles in the transfer function that tends the system to be sensitive to perturbations again. Aiming at delay cancellation, inverse of Pade approximation should be cascaded to velocity output for prediction; however, note that (27) is a nonminimum phase system hence its inverse is unstable. Therefore, another rational function approximation with zero numerator dynamics is used by taking first $n$-terms of Maclaurin series (Vajta, 2000). Although this approach provides a coarse approximation compared to standard Pade approximation, it is implementable. In this case, (27) and (28) become

$$
\begin{gathered}
e^{-T_{d} s} \approx R(s)=\frac{1}{\sum_{k=0}^{n} c_{k} s^{k}} \\
c_{k}=\frac{T_{d}^{k}}{k !}, k=0,1, \ldots, n .
\end{gathered}
$$

Then, for an $n^{\text {th }}$ order velocity prediction, it is sufficient to multiply velocity measurement with

$$
R(s)^{-1} \approx 1+T_{d} s+\frac{T_{d}^{2}}{2} s^{2}+. .+\frac{T_{d}^{n}}{n !} s^{n},
$$

Consequently, it is observed that either inverse of first order Pade approximation or Euler method can perform adequately for velocity prediction when dead time is not large compared with the bandwidth of the system. The general representation of an $n^{\text {th }}$ order predictor is illustrated in Figure 3. Note that both first order predictors have the same structure. Particularly, Euler method and inverse Pade approximant have the same structure as in Figure 3 with different $c_{1}$ coefficients.

Since the measured velocity $v_{o}(t)$ is the delayed velocity of the actual velocity $v(t)$, we can only use the estimated velocity $\hat{v}(t)$ in the observer equations given by (3)-(4). In this case, the observer should be given as follows

$$
\dot{z}=g^{\prime}(\hat{v}) \frac{u}{M}
$$

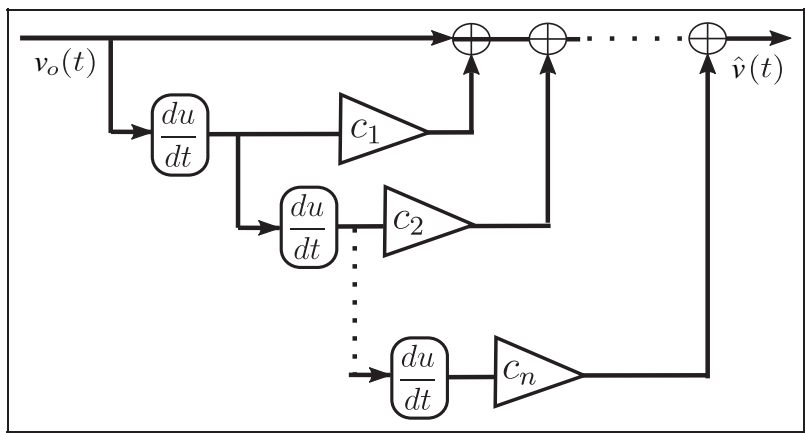

Figure 3. General structure of an $\mathrm{n}$-th order predictor.

$$
\hat{a}_{c}=z-g(\hat{v})
$$

Then, the parameter estimation error $e(t)=a_{c}-\hat{a}_{c}$ dynamics is given as

$$
\dot{e}=-g^{\prime}(\hat{v}) \frac{u}{M}+g^{\prime}(\hat{v}) \dot{\hat{v}}
$$

Defining the velocity estimation error $e_{v}$ as given by (20), by using (32)-(33) we obtain

$$
\begin{aligned}
\dot{e}= & g^{\prime}(\hat{v})\left(\hat{a}_{c} \operatorname{sgn}(\hat{v})-a_{c} \operatorname{sgn}(v)\right)-g^{\prime}(\hat{v}) \dot{e}_{v} \\
= & -g^{\prime}(\hat{v}) \operatorname{sgn}(\hat{v}) e+g^{\prime}(\hat{v}) a_{c}(\operatorname{sgn}(\hat{v})-\operatorname{sgn}(v)) \\
& -g^{\prime}(\hat{v}) \dot{e}_{v} .
\end{aligned}
$$

If $\operatorname{sgn}(\hat{v})=\operatorname{sgn}(v)$, which is satisfied when $|v|>\left|e_{v}\right|$, then the parameter error dynamics becomes

$$
\dot{e}=-g^{\prime}(\hat{v}) \operatorname{sgn}(\hat{v}) e-g^{\prime}(\hat{v}) \dot{e}_{v} .
$$

Note that with velocity estimation error $e_{v}$ satisfying $e_{v}=0$, we have $v=\hat{v}$, and (36) becomes the same as (6). When $e_{v} \neq 0$, the perturbation term $g^{\prime}(\hat{v}) \dot{e}_{v}$ could be considered as a disturbance acting on an exponentially stable system. Since exponentially stable systems are robust to perturbations, we could expect stable (may not be necessarily asymptotically stable) error dynamics in (36). Consequently, in the presence of time delay, when the conditions stated in Lemma (2) are satisfied and velocity error and time delay are small enough, it is expected to have stable error dynamics as in delay free design. The following lemma clarifies this point.

Lemma 3: Consider the system given by (1)-(2). Assume that $\hat{v}$ is the estimation of $v(t)$ (see Figure 1) and that the observer is given as (32)-(33), where $g(v): \mathbf{R} \mapsto \mathbf{R}$ satisfies the conditions stated in Lemma 1 (or Lemma 2). Furthermore, assume that $\operatorname{sign}(\hat{v})=\operatorname{sign}(v)$. Under these conditions, if $\left|g^{\prime}(\hat{v}) \dot{e}_{v}\right|<m$ for some $m>0$, then the parameter estimation error $e$ given by (6) is bounded as well. Furthermore, if $m \rightarrow 0$, then $|e| \rightarrow 0$ as well.

Proof: Note that when $e_{v}=0$, that is, $\hat{v}(t)=v(t)$, then the parameter error satisfies (6), which is shown to be exponentially stable in Lemma 1 or Lemma 2 . Then, by classical converse theorems of Lyapunov stability theory, there exists a 
Lyapunov function $V: \mathbf{R}_{+} \times R \mapsto \mathbf{R}_{+}$that satisfies the following

$$
\begin{aligned}
n_{1}\|e\|^{2} \leqslant V(t, e) & \leqslant n_{2}\|e\|^{2} \\
\frac{\partial V}{\partial t}+\frac{\partial V}{\partial e}\left[-g^{\prime}(\hat{v}) \operatorname{sgn}(\hat{v}) e\right] & \leqslant-n_{3}\|e\|^{2} \\
\left\|\frac{\partial V}{\partial e}\right\| & \leqslant n_{4}\|e\|
\end{aligned}
$$

for some positive constants $n_{1}, n_{2},, n_{3}, n_{4}$, see Khalil (2002). By using this function from (36), we obtain

$$
\begin{aligned}
\dot{V}(t, e) & =\frac{\partial V}{\partial t}+\frac{\partial V}{\partial e}\left[-g^{\prime}(\hat{v}) \operatorname{sgn}(\hat{v}) e\right]-\frac{\partial V}{\partial e}\left(g^{\prime}(\hat{v}) \dot{e}_{v}\right) \\
& \leqslant-n_{3}\|e\|^{2}+n_{4}\|e\| m \\
& \leqslant-n_{3}\|e\|\left(\|e\|-\frac{n_{4}}{n_{3}} m\right)
\end{aligned}
$$

clearly, if $\|e\| \geqslant \frac{n_{4}}{n_{3}} m$, then $\dot{V}<0$ and by (37), $\|e\|$ is bounded. By using standard Lyapunov Theory arguments it follows that the error is bounded (Khalil, 2002). In fact, $\lim _{t \rightarrow \infty}\|e(t)\|<\frac{n_{4}}{n_{3}} m$. Clearly, as $m \rightarrow 0$, we have $e \rightarrow 0$ as well.

Remark 4: Lemma 1 and 2 show that when the measurements are not delayed (i.e. $T_{d}=0$ in Figure 1), the observer structure given by (3)-(4) achieves exact friction cancellation since the parameter error dynamics is exponentially stable. When there is a measurement delay (i.e. $T_{d}>0$ in Figure 1), obviously we need to estimate $v(t)$ for exact friction cancellation. In such a case, we need a method to estimate $v(t)$ based on the delayed measurements $v_{o}(t)$. If the methodology chosen for such an estimation yields small errors, then Lemma 3 guarantees that the friction parameter estimation error also remains small. If for such an estimation we choose Euler approximation as given by (21)-(22), we can obtain the velocity estimation error bounds. Note that Taylor series expansion of a function $f: \mathbf{R} \mapsto \mathbf{R}$ can be given as follows

$$
f\left(t+T_{d}\right)=f(t)+T_{d} \dot{f}(t)+\frac{1}{2} T_{d}^{2} \ddot{f}(\xi)
$$

where $t<\xi<t+T_{d}$, see Atkinson (1989). Now, if we choose $f(t)=v_{o}(t)$ in (43), we obtain

$$
v_{o}\left(t+T_{d}\right)=v_{o}(t)+T_{d} \dot{v}_{o}(t)+\frac{1}{2} T_{d}^{2} \ddot{v}_{o}(\xi)
$$

where $t<\xi<t+T_{d}$. We note that the last term in (44) is called as the discretization or truncation error of Euler Method (Atkinson, 1989). By using (19) and (23) in (44), we obtain

$$
v(t)=\hat{v}(t)+\frac{1}{2} T_{d}^{2} \ddot{v}_{o}(\xi)
$$

By using (20), we easily obtain

$$
\left|e_{v}\right|=|v(t)-\hat{v}(t)| \leqslant \frac{1}{2} T_{d}^{2} \hat{m}_{1}
$$

where $\hat{m}_{1}=\max \left|\ddot{v}_{o}(t)\right|$. Similarly, if we use $f(t)=\dot{v}_{o}(t)$ in (43) we obtain ${ }^{t}$

$$
\left|\dot{e}_{v}\right|<\frac{1}{2} T_{d}^{2} \hat{m}_{2}
$$

where $\hat{m}_{2}=\max \left|\dddot{v}_{o}(t)\right|$ Clearly, if $T_{d}$ is sufficiently small, then the conditions of Lemma 3 are satisfied and subsequently the performance of the proposed friction compensation scheme will be satisfactory. Obviously, better velocity estimation schemes will yield better friction compensation performance. In the present paper, we will consider only some basic velocity estimation schemes such as the classical Euler method mentioned above. However, better estimation schemes that yield better velocity estimation error bounds require and deserve further research.

\section{Smith predictor-based velocity controller design}

As stated by Taşdelen and Özbay (2013), it is possible to design a Smith predictor-based controller using controller parametrization method given in Youla et al. (1976) for the required robustness and performance objectives. Hence, although it is possible to extend the idea in Taşdelen (2013) for different transfer functions, we can simply define transfer function of the plant with dead time as

$$
P(s)=\frac{1}{M s} e^{-T_{d} s}
$$

In fact, if a gain block $\mathrm{M}$ is appended to velocity controller, $C_{v}(s)$ illustrated in Figure 4, the transfer function becomes simpler such that $P_{0}(s)=\frac{1}{s} e^{-T_{d} s}$. Afterwards, it is sufficient to design an internal controller $C_{0 v}(s)$ to stabilize the velocity loop. Finally, complete controller $C_{v}(s)$, illustrated in Figure 4 , can be obtained as

$$
C_{v}(s)=\frac{C_{0 v}(s)}{1+C_{0 v}(s)\left(\frac{1-e^{-T_{d} s}}{s}\right)}
$$

Therefore, $T_{0 v}(s)$ which is closed loop transfer function of velocity loop formed by $\left(C_{v}(s), P(s)\right)$ can be written as

$$
T_{v}(s)=\frac{C_{0 v}(s)}{s+C_{0 v}(s)} e^{-T_{d} s}
$$

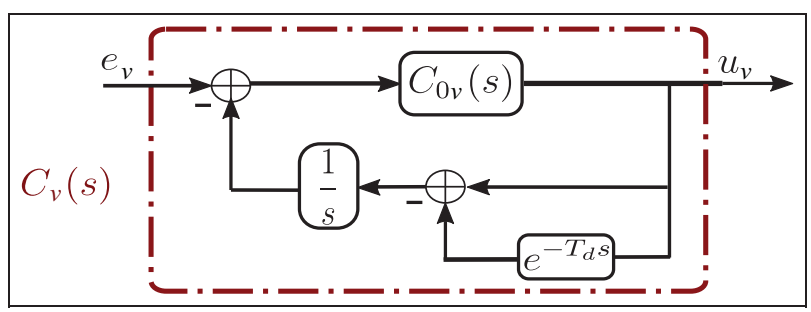

Figure 4. Smith predictor-based velocity controller. 
Note that it is also possible to decompose (50) into

$$
T_{v}(s)=T_{0 v}(s) e^{-T_{d} s}
$$

where $T_{0 v(s)}=C_{o v}(s) /\left(s+C_{0 v}(s)\right)$ is delay free closed loop velocity transfer function.

To design an internal controller, controller parametrization method, which is explained step by step as below, should be utilized:

- Transfer function of the delay free plant should be written in terms of coprime, rational and stable functions $N_{p}(s)$ and $D_{p}(s)$ such that

$$
P_{0}(s)=\frac{N_{p}(s)}{D_{p}(s)} .
$$

- Let chosen $D_{p}(s)$ have zeros at $s=z_{1}, z_{2}, \ldots, z_{n}$. Then, another stable function $X(s)$ which satisfies $X\left(z_{1}\right)=1 / N_{p}\left(z_{1}\right), X\left(z_{2}\right)=1 / N_{p}\left(z_{2}\right), \ldots, X\left(z_{n}\right)=1 / N_{p}\left(z_{n}\right)$ should be chosen.

- Lastly, a stable $Y(s)$ can be found by solving the Bezout equation given below

$$
X(s) N_{p}(s)+Y(s) D_{p}(s)=1 .
$$

- Using these functions, the set of all stabilizing controllers can be written as

$$
C(s)=\frac{X(s)+D_{p}(s) Q(s)}{Y(s)-N_{p}(s) Q(s)} .
$$

where $Q(s)$ is proper and stable function satisfying $Q(s) \neq Y(s) N_{p}(s)^{-1}$. Also, it should be selected based on desired performance and robustness criterion; therefore, the minimum degree of $Q(s)$ is one less than the number of interpolation conditions. For instance, in order to obtain minimum degree controller stabilizing $P_{0}(s)$ when step input is applied, a particular choice might be $Q(s)=0$ since plant has already a pole at $s=0$. Then, for $N_{p}(s)=1 /\left(s+K_{v}\right)$, $D_{p}(s)=s /\left(s+K_{v}\right), X(s)=K_{v}$ and $Y(s)=1$, a possible internal velocity controller, $C_{0 v}(s)$, can be designed such that

$$
C_{0 v}(s)=K_{v}
$$

where $K_{v}$ is a free design parameter which will be determined by pole placement method. Then, as in Odabas (2014), $C_{v}(s)$, the complete form of Smith predictor-based controller and $T_{v}(s)$, closed loop transfer function for velocity, can be written as

$$
\begin{aligned}
C_{v}(s) & =\frac{K_{v}}{1+K_{v}\left(\frac{1-e^{-T_{d} s}}{s}\right)}, \\
T_{v}(s) & =\frac{K_{v}}{s+K_{v}} e^{-T_{d} s} .
\end{aligned}
$$

However, if ramp input tracking is desired, $Q(0)=Y(0) / N(0)$ condition should be satisfied as well. In this case, we can simply choose $Q(s)=K_{v}$. Then, transfer function of ramp tracking Smith predictor-based controller and closed loop system becomes

$$
\begin{aligned}
C_{0 v}(s) & =\frac{2 K_{v}+K_{v}^{2} s}{s}, \\
C_{v}(s) & =\frac{2 s^{2} K_{v}+s K_{v}^{2}}{s^{2}+\left(2 K_{v} s+K_{v}^{2}\right)\left(1-e^{-T_{d} s}\right)}, \\
T_{v}(s) & =\frac{2 K_{v} s+K_{v}^{2}}{\left(s+K_{v}\right)^{2}} e^{-T_{d} s} .
\end{aligned}
$$

To sum up, it is possible to change controller design to satisfy desired robustness and performance requirements by simply changing $Q(s)$ and pole locations of the controller. Note that for this paper, the same controller design procedure given in Odabaş and Morgül (2014) is utilized. In other words, although modeling of dead time as actuator or measurement delay alters observer design, it does not change Smith predictor-based controller design for closed loop system.

\section{Smith predictor-based position controller design}

Smith predictor-based position controller has a very similar structure to velocity controller given in the previous section. Distinctively, position controller, $C_{p}$, includes delay free transfer function of the velocity closed loop, $T_{0 v}$, as in Figure 5. Thus, once the velocity controller is designed, the same design procedure can be implemented for a position loop extension as well (Odabaş, 2014). For step input tracking case, closed loop pole $K_{p}$ can be located by again using pole placement method. Thus, transfer function of the controller can be given as

$$
\begin{aligned}
C_{0 p}(s) & =K_{p} . \\
C_{p}(s) & =\frac{K_{p}}{1+K_{p}\left(\frac{1-T_{0 v}(s) e^{-T_{d} s}}{s}\right)} .
\end{aligned}
$$

Then, closed loop transfer function of hierarchical position system can be obtained as follows

$$
\begin{aligned}
T_{p}(s) & =\frac{K_{p}}{s+K_{p}} T_{v}(s) \\
& =\frac{K_{p}}{\left(s+K_{p}\right)} \frac{K_{v}}{\left(s+K_{v}\right)} e^{-T_{d} s} .
\end{aligned}
$$

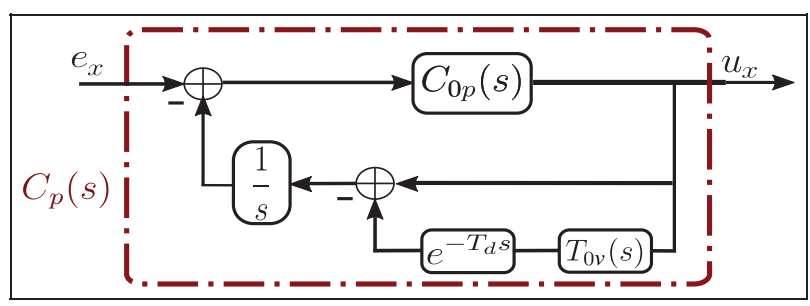

Figure 5. Smith predictor-based position controller. 
Similarly, after ramp input tracking extension of velocity loop given by (58) and (60), we obtain the following transfer functions for the position loop

$$
\begin{aligned}
C_{0 p}(s) & =\frac{2 K_{p} s+K_{p}^{2}}{s}, \\
C_{p}(s) & =\frac{2 K_{p} s^{2}+K_{p}^{2} s}{s^{2}+\left(2 K_{p} s+K_{p} 2\right)\left(1-T_{0 v}(s) e^{-T_{d} s}\right)}, \\
T_{p}(s) & =\left(\frac{2 K_{p} s+K_{p}^{2}}{s^{2}+2 K_{p} s+K_{p}^{2}}\right) T_{v}(s) .
\end{aligned}
$$

Mathematically, $K_{v}$ and $K_{p}$ can be set freely according to desired closed loop transfer function of the system. However, intuitively, it is generally expected that inner loop poles will be larger compared with outer loop poles in the literature. Therefore, although it is not a strict requirement, poles of (63) or (66) may be allocated such that $K_{v}>K_{p}$.

In such a position control system, time delay can be revealed by considering system properties or inferred after some system identification techniques. Although it is out of main context in this paper, interested readers can find lots of interesting papers in the literature regarding this point (Uyanik et al., 2015; Wang and Zhang, 2001) Likewise, robustness to time delay perturbations is analyzed by calculating vector margin in Taşdelen (2013). Assuming that friction cancellation strategy presented in this paper is achieved well enough, it can be concluded that small mismatches between actual and inferred time delays can affect the robustness of the controllers only slightly.

\section{Reference filter design}

The reference filter in Figure 1 is employed to enhance the tracking performance of the system by suppressing higher order dynamics. Basically, a stable and strictly proper first order reference filter $H(s)$, with a unit gain such that $H(0)=1$ can be incorporated so that existence of the reference filter has no effect on the transfer function from disturbance to output. One particular choice is

$$
H_{p}(s)=\frac{1}{1+s / w_{p c}} .
$$

where cut-off frequency, $w_{p c}$, is a free design parameter. Smaller cut-off frequency provides smaller overshoots. However, diminution of the cut-off frequency affects the rising time negatively. In other words, the system response starts to slow down, that is why $w_{p c}$ should be chosen according to the design requirements on overshoot and rise time of the system. Typically, it is chosen to cancel the fastest negative real axis zero of closed loop system. To this end, generally, first order designs are employed in the literature, since higher order filters incorporate extra lags on the input signal and can cause undesired distortions on input waveform. Certainly, more complex filter designs might be implemented as well to improve set-point responses considering this trade-off. As an
Table I. Parameters used in simulations.

\begin{tabular}{lll}
\hline Parameter & Notation & value \\
\hline Stribeck velocity & $v_{s}$ & $0.001 \mathrm{~m} / \mathrm{s}$ \\
Stiffness coefficient & $\sigma_{0}$ & $10^{5} \mathrm{~N} / \mathrm{m}$ \\
Damping coefficient & $\sigma_{l}$ & $\sqrt{10^{5}} \mathrm{Ns} / \mathrm{m}$ \\
Coulomb friction & $F_{c}$ & $4 \mathrm{~N}$ \\
Stick friction & $F_{s}$ & $4.7 \mathrm{~N}$ \\
Viscous friction & $F_{v}$ & $0.4 \mathrm{Ns} / \mathrm{m}$ \\
Total mass & $M$ & $5 \mathrm{~kg}$ \\
Position controller design parameter & $K_{p}$ & \\
$\quad$ Triangular wave tracking & & 4 \\
$\quad$ Square wave tracking & & 5 \\
Velocity controller design parameter & $K_{v}$ & \\
$\quad$ Triangular wave tracking & & 20 \\
$\quad$ Square wave tracking & & 15 \\
Dead time & $T_{d}$ & $0.1 \mathrm{~s}$ \\
Reference filter cutoff freq. & $W_{p c}$ & $3 \mathrm{rad} / \mathrm{s}$ \\
Derivative filter coefficient & $N$ & 100 \\
Observer estimation function & $g(\hat{v})$ & $\mathrm{k} . \mathrm{In}(\cosh (\hat{\mathrm{v}}))$ \\
& $g(\hat{v})$ & $\mathrm{k} . \tanh (\hat{\mathrm{v}})$ \\
\hline
\end{tabular}

example, Feliu-Batlle and Rivas-Perez (2019) insert inverse of plant model inside the transfer function of filter.

\section{Simulation results}

We performed various simulations for the system given in Figure 1 with the parameters specified in Table 1. For the simulations, we used Matlab Simulink with ode4 solver, which utilizes $4^{\text {th }}$ order Runge-Kutta formula, and for the friction we used Coulomb and viscous friction block of Simulink, by setting the viscous friction coefficient to zero. Note that Coulomb friction is modeled by discontinuous sign function, which may cause some undesired effects under certain conditions (see Remark 5 at the end of this section).

In the first set of simulations, whose results are given in Figures 6-9, we assumed that only Coulomb friction is present with friction constant, as indicated in Table 1. To see the effect of our proposed friction and velocity estimator, we first considered unit step tracking response in the position loop by using first order Pade approximant for the velocity estimator. As can be seen in Figure 6, when the actual velocity is known, the friction parameter estimation error $e$ converges to zero when the observer gain is selected as $k=5$ or $k=10$. We note that this behavior is in agreement with the conclusions given in Lemma 1 and Remark 2. For the same observer gains, when the actual velocity is predicted with the proposed first order Pade approximant, a similar convergence behavior is observed. As it can be seen in Figure 6, while in both cases the steady-state behavior of $e(t)$ is similar, the transient response (e.g. for $t<1.5 \mathrm{sec}$.) when velocity predictor is utilized may exhibit some relatively large overshoots especially when the observer gain is increased.

The effect of observer gain $k$ on the velocity prediction error $e_{v}$ is given in Figure 7. Similar to the observation given above, while $e_{v}$ converges to zero, the transient response (e.g. 


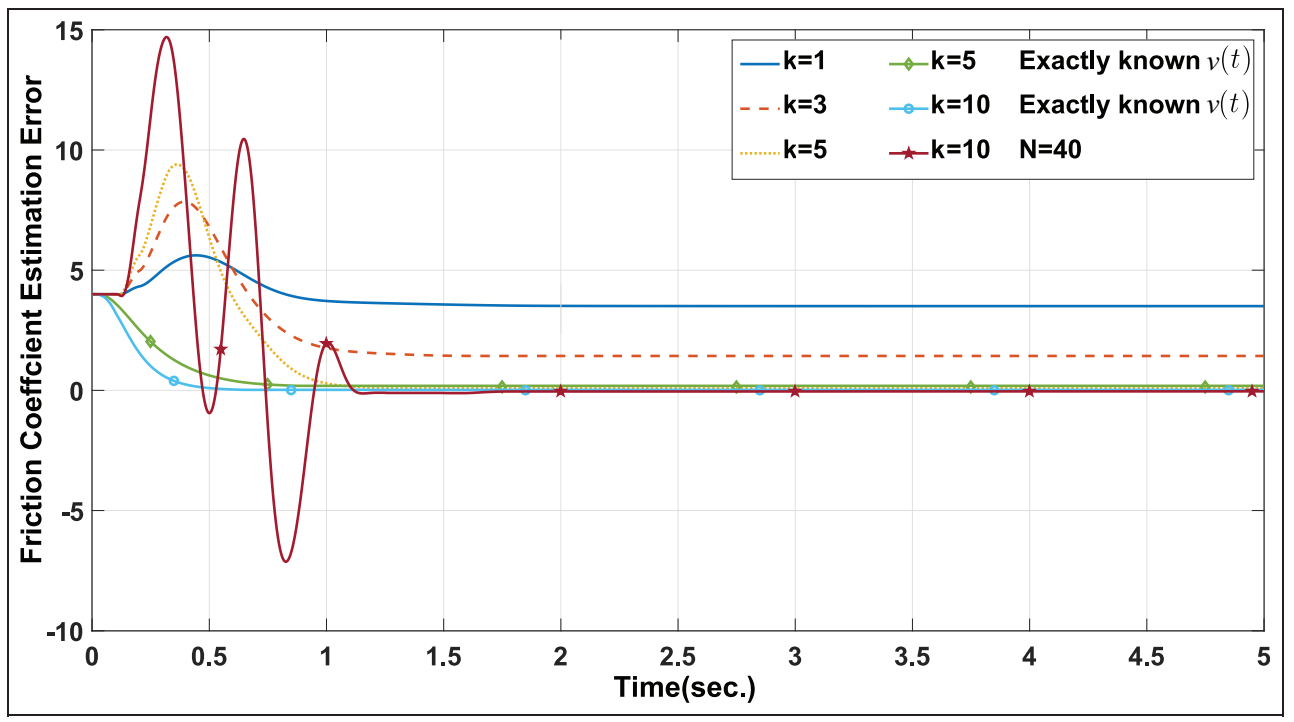

Figure 6. Friction estimation error, e, for unit step position tracking response with $\mathrm{I}^{\text {st }}$ order inverse Pade approximant velocity prediction under Coulomb friction.

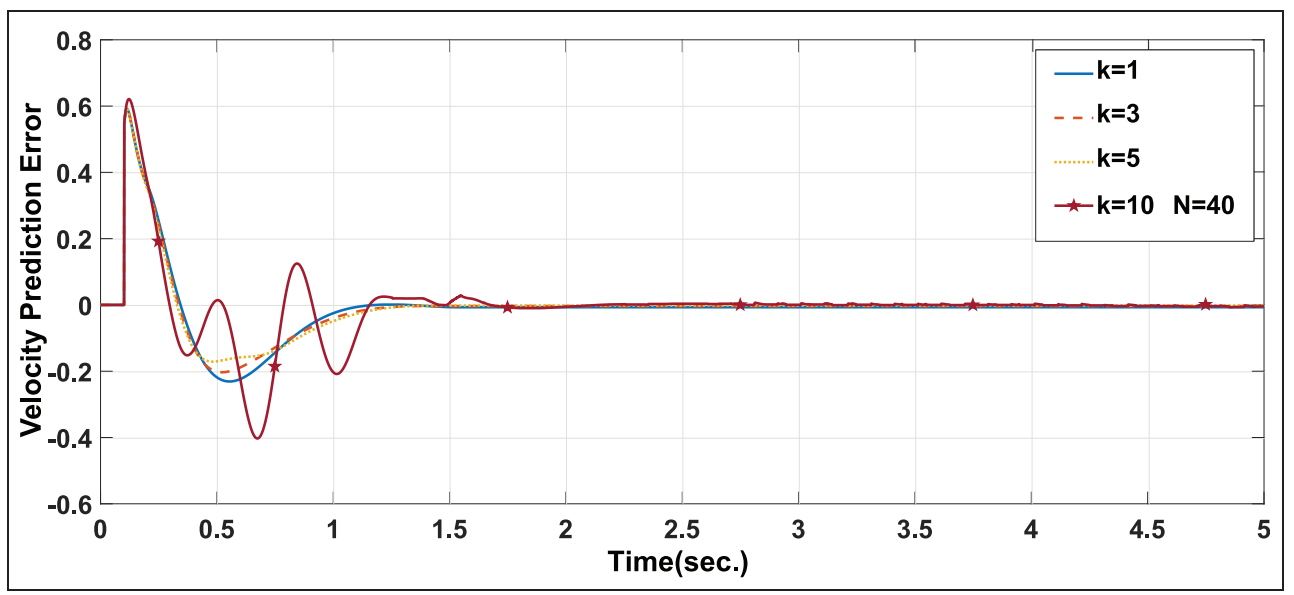

Figure 7. Velocity prediction error, $e_{v l}$, for unit step position tracking response with $\left.\right|^{\text {st }}$ order inverse Pade approximant velocity prediction under Coulomb friction.

for $t<1.5 \mathrm{sec}$.) may show some relatively large overshoots, especially for large observer gains.

We note that as the observer gain $k$ increases, the convergence rate of friction estimation error also increases when the actual velocity is available, see Figure 6. This observation is also in agreement with Remarks 2 and 3. On the other hand, when the observer gain is too low, the friction estimation error may not converge to zero within a reasonable duration. When the actual velocity is not known and should be estimated, we observe that initial fluctuations on the velocity prediction errors may cause some undesired transient effects on the friction estimation error for large observer gains (see Figure 6). Furthermore, the last observation may also apply to the case of large time delays, since naturally one expects a decrease in the velocity prediction performance. Hence, selection of optimal observer gain appears to be an important problem that requires further investigation. In this respect, we also observed that decreasing $N$, cut-off frequency of the low-pass filtered derivative in velocity prediction block, may also improve the transient response even for relatively large observer gains, and hence may enhance both friction and velocity estimation performances. This approach may also be beneficial under the presence of dynamic friction (see e.g. the results given in Tables 2 and 3). Hence, the relation between $k$ and $N$ also requires further study.

Additionally, square and triangular wave input tracking performances of the system are investigated again with first order Pade approximant based velocity prediction. In the simulations whose results are given in Figures 8 and 9, we still use only Coulomb friction with $F_{c}=4$. Exclusively, for ramp 


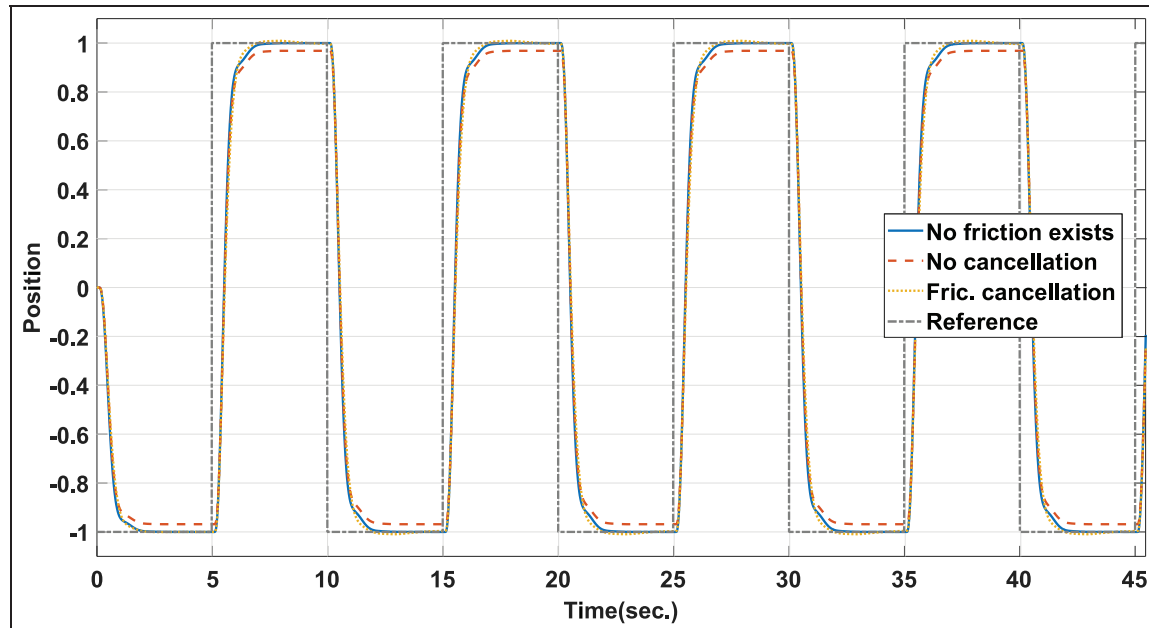

(a)

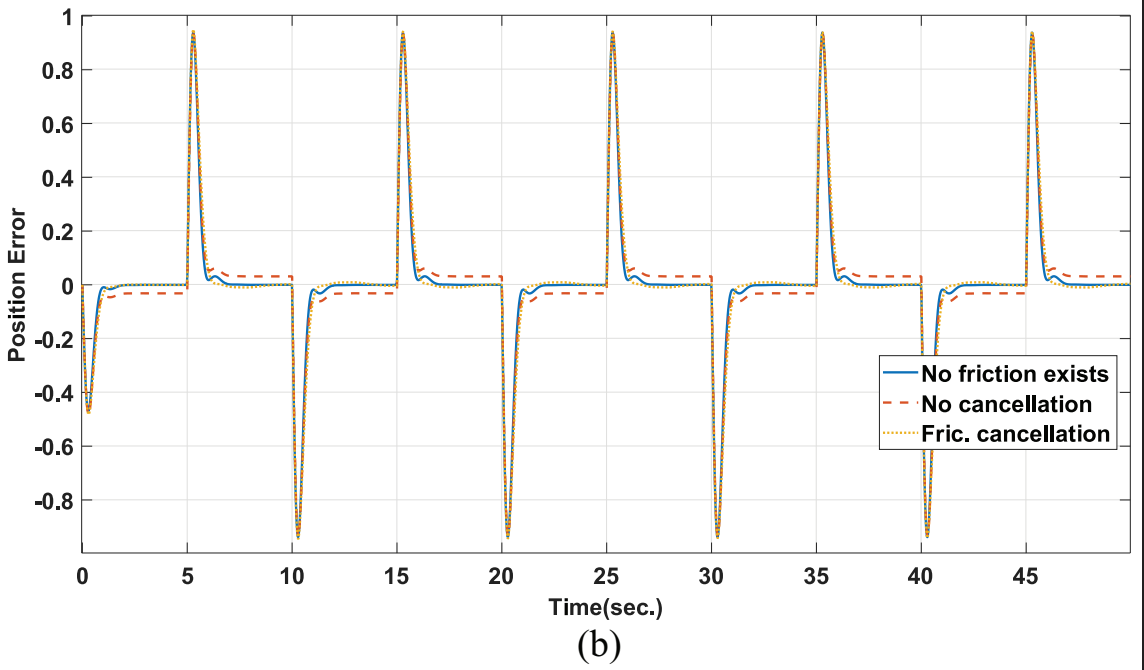

Figure 8. System response for unit square wave input with $I^{\text {st }}$ order inverse Pade approximant-based velocity prediction when $k=5$ and $T_{d}=0.1$. Without Coulomb friction compensation, steady state values for positive and negative position response are $x_{s s}^{+}=0.968$ and $x_{s s}^{-}=-0.968$ respectively. (a) Position tracking performance (b) Position tracking error.

input tracking case, reference filter can be removed to enhance the performance since velocity dynamics change slow enough already. Position responses show that observer based cancellation improves the performance when observer gain is $k=5$ and time delay is $T_{d}=0.1$. Thus, it can be concluded that deployed adaptive observer and velocity predictor can eliminate the friction adequately and ensure a comparable performance to no friction case. Otherwise, Smith predictor-based controllers can partially extinguish the position error therefore; a steady state error occurs as in Figures 8 and 9. Besides, Coulomb friction cancellation performances of different velocity prediction schemes are presented in Table 2 for unit step tracking. From the table, it can be observed that closed loop performances increase with the proposed observer and velocity predictions, as it is expected.

Finally, in Friedland and Mentzelopoulou (1992), it is claimed that original observer design is able to estimate friction terms which are not hindered to Coulomb coefficient that is why we performed several simulations in addition to the ones given above. During these additional simulations, LuGre model, a well known and frequently used dynamic model in the literature, is employed to mimic real friction force illustrated as friction block in Figure 1 such that

$$
\begin{gathered}
\dot{z}_{d}=v-\sigma_{0} \frac{|v|}{h(v)} z_{d}, \\
F(v)=\sigma_{0} z_{d}+\sigma_{1}(v) \dot{z}_{d}+F_{v} v
\end{gathered}
$$

where $z_{d}$ represents the internal state of the LuGre friction model, $h(\cdot)$ is an appropriately defined function to capture Stribeck effect, Coulomb and stick friction and can be written as

$$
h(v)=F_{c}+\left(F_{s}-F_{c}\right) e^{-\left(v / v_{s}\right)^{2}} .
$$




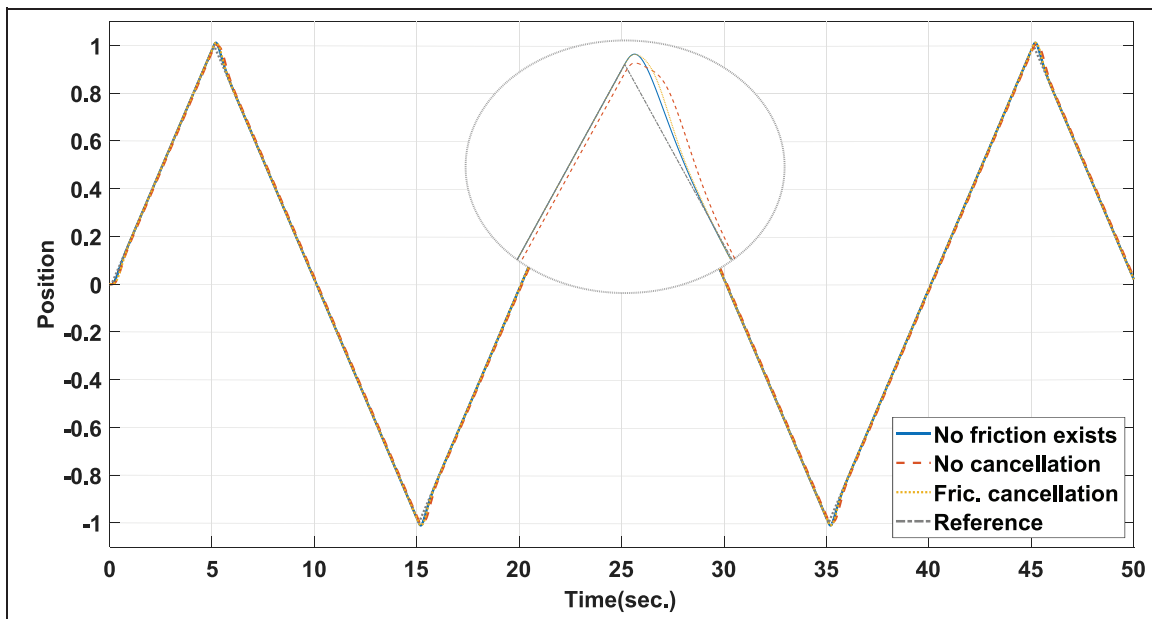

(a)

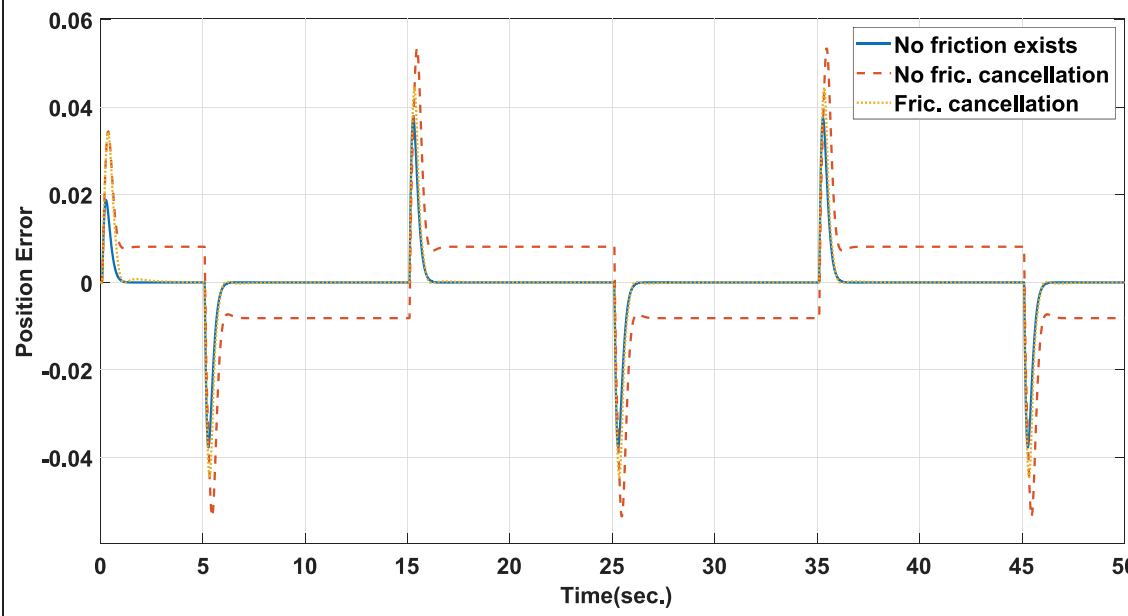

(b)

Figure 9. System response for unit triangular wave input with $\left.\right|^{\text {st }}$ order inverse Pade approximant-based velocity prediction when $k=5$ and $T_{d}=0.1$. Without Coulomb friction compensation, steady state errors for positive and negative position response are $\mathrm{e}_{s s}^{+}=0.8 \%$ and $\mathrm{e}_{s s}^{-}=-0.8 \%$ respectively. (a) Position tracking performance (b) Position tracking error.

For the meaning of various coefficients given in (68)-(69), see Table 1 . Note that the steady-state relation between velocity and friction force for LuGre model is obtained for $\dot{z}_{d}=0$. In this case, (69) becomes

$$
\begin{aligned}
F(v) & =h(v) \operatorname{sgn}(v)+F_{v}(v) \\
& =\left[F_{c}+\left(F_{s}-F_{c}\right) e^{-\left(v / v_{s}\right)^{2}}\right] \operatorname{sgn}(v)+F_{v} v .
\end{aligned}
$$

In Table 3, rise time, settling time and steady state values are compared for different time delay and prediction method combinations for step input tracking with LuGre Friction model. Clearly, for a fixed $k$, it is required to apply filtering with lower cut off frequencies to obtain similar position tracking errors when either observer gain or dead time increases. Certainly, in all cases, proposed adaptive observer plus velocity predictor structure improves system performance. Among these experiments, the best performance is achieved when actual velocity can be instantaneously measured; however, in the scope of this paper, this is not possible due to time delay considered in measurement process. This case is investigated just to verify that if we can predict velocity values so close to actual velocity values, we can obtain friction cancellation similar to delay free measurement. Furthermore, it is obvious that when there is no friction compensation, the system suffers from performance degradation gradually as time delay increases. It seems that all prediction methods can enhance set point responses to some extend. Second order methods, both Pade and Heun, provide similar results and outperform the first order approximations for same observer gains. However, when there exists large time delay, they become more sensitive to velocity changes than Euler and inverse first order Pade approximant that is why it is necessary to design a derivative filter with a lower cut-off to obtain appropriate responses. As a last observation, it can be said that although they have similar structure (see Figure 3), Euler approximation provides a faster friction but more gain sensitive estimation compared to inverse first order Pade 
Table 2. Comparison of different velocity prediction approaches for $T_{d}=0.1 / 0.2 / 0.3 \mathrm{sec}$. under Coulomb friction modeling. $(N=100$ in general except ${ }^{\bullet}: N=$ I).

\begin{tabular}{|c|c|c|c|c|c|c|c|c|c|}
\hline & Rise & Time & (sec.) & Settling & Time & (sec.) & Steady & State & Value \\
\hline No friction exists & 0.57 & 0.57 & 0.60 & 1.18 & 1.18 & 1.23 & 1.00 & 1.00 & 1.00 \\
\hline No friction cancellation & 0.61 & 0.62 & 0.66 & 0.67 & 2.19 & 1.47 & 0.97 & 0.94 & 0.89 \\
\hline \multicolumn{10}{|l|}{ Observer gain $k=1$} \\
\hline Cancellation with exactly known $v(t)$ & 0.60 & 0.62 & 0.70 & 1.68 & 1.74 & 1.44 & 0.99 & 0.97 & 0.95 \\
\hline Cancellation without prediction & 0.68 & 0.83 & 1.10 & 1.83 & 2.00 & 2.18 & 0.98 & 0.93 & 0.85 \\
\hline Euler approximation & 0.65 & 0.74 & 1.10 & 12.04 & 3.05 & 3.72 & 0.98 & 0.95 & 0.92 \\
\hline $1^{\text {st }}$ order Pade inverse & 0.69 & 0.76 & $0.72^{\circ}$ & 1.84 & 2.28 & $3.31^{\circ}$ & 0.98 & 0.94 & $0.89^{\circ}$ \\
\hline Heun's method & 0.60 & 0.71 & $1.36^{\circ}$ & 1.70 & 4.33 & $3.34^{\circ}$ & 0.99 & 0.96 & $0.94^{\circ}$ \\
\hline $2^{\text {nd }}$ order Pade inverse & 0.60 & 0.71 & $1.36^{\circ}$ & 1.71 & 4.33 & $3.34^{\circ}$ & 0.99 & 0.95 & $0.94^{\circ}$ \\
\hline \multicolumn{10}{|l|}{ Observer gain $k=5$} \\
\hline Cancellation with exactly known $v(t)$ & 0.60 & 0.58 & 0.59 & 1.57 & 1.19 & 1.31 & 1.00 & 1.00 & 1.00 \\
\hline Cancellation without prediction & 0.74 & 1.06 & 1.34 & 1.82 & 1.66 & 2.10 & 1.00 & 0.97 & 0.95 \\
\hline Euler approximation & 0.60 & 1.06 & 0.94 & 1.67 & 2.02 & $4.56^{\circ}$ & 1.00 & 1.00 & $1.00^{\circ}$ \\
\hline$\left.\right|^{\text {st }}$ order Pade inverse & 0.66 & 0.85 & $1.45^{\circ}$ & 1.50 & 1.68 & $2.24^{\circ}$ & 1.00 & 0.99 & $0.98^{\circ}$ \\
\hline Heun's method & 0.57 & $1.22^{\circ}$ & $1.48^{\circ}$ & 2.35 & $1.95^{\circ}$ & $2.25^{\circ}$ & 1.00 & $1.00^{\circ}$ & $0.98^{\circ}$ \\
\hline $2^{\text {nd }}$ order Pade inverse & 0.57 & $1.22^{\circ}$ & $1.47^{\circ}$ & 2.35 & $1.95^{\circ}$ & $2.24^{\circ}$ & 1.00 & $1.00^{\circ}$ & $0.98^{\circ}$ \\
\hline
\end{tabular}

Table 3. Comparison of different velocity prediction approaches for $T_{d}=0.1 / 0.2 / 0.3 \mathrm{sec}$. under LuGre Friction modeling. $(N=100$ in general except $^{\star}: N=10, \bullet: N=1$ and ${ }^{\dagger}: N=0.3$ ).

\begin{tabular}{|c|c|c|c|c|c|c|c|c|c|}
\hline & Rise & Time & (sec.) & Settling & Time & (sec.) & Steady & State & Value \\
\hline No friction exists & 0.57 & 0.57 & 0.60 & 1.18 & 1.18 & 1.23 & 1.00 & 1.00 & 1.00 \\
\hline No friction cancellation & 0.59 & 0.62 & 0.66 & 1.55 & 1.32 & 1.28 & 0.96 & 0.93 & 0.87 \\
\hline \multicolumn{10}{|l|}{ Observer gain $k=1$} \\
\hline Cancellation with exactly known $v(t)$ & 0.63 & 0.69 & 0.84 & 1.90 & 7.79 & 16.38 & 1.00 & 1.00 & 1.00 \\
\hline Cancellation without prediction & 0.67 & 1.08 & 1.45 & 1.70 & 23.48 & 26.99 & 0.98 & 0.96 & 0.90 \\
\hline Euler approximation & 0.66 & 0.81 & $1.7 \mid$ & 2.30 & 16.69 & 31.48 & 1.00 & 0.98 & 0.96 \\
\hline $1^{\text {st }}$ order Pade inverse & 0.69 & 0.78 & $1.80^{\star}$ & 4.37 & 2.22 & $27.48^{\star}$ & 1.00 & 0.95 & $0.96^{\star}$ \\
\hline Heun's method & 0.63 & 0.78 & $1.5 \mathrm{I}^{\bullet}$ & 1.98 & 16.96 & $29.91^{\bullet}$ & 1.00 & 0.98 & $0.96^{\circ}$ \\
\hline $2^{\text {nd }}$ order Pade inverse & 0.63 & 0.78 & $1.51^{\bullet}$ & 1.98 & 16.96 & $29.91^{\bullet}$ & 1.00 & 0.98 & $0.96^{\circ}$ \\
\hline \multicolumn{10}{|l|}{ Observer gain $k=3$} \\
\hline Cancellation with exactly known $v(t)$ & 0.60 & 0.63 & 0.71 & 1.59 & 1.29 & $1.4 \mathrm{I}$ & 1.00 & 1.00 & 1.00 \\
\hline Cancellation without prediction & 0.76 & 1.06 & 1.81 & 1.79 & 14.07 & 32.05 & 1.00 & 0.98 & 0.95 \\
\hline Euler approximation & 0.62 & $0.95^{\star}$ & $2.00^{\dagger}$ & 1.52 & $|4.8|^{\star}$ & $7.07^{\dagger}$ & 1.00 & $0.99^{\star}$ & $1.00^{\dagger}$ \\
\hline $\mathrm{I}^{\text {st }}$ order Pade inverse & 0.69 & 0.87 & $2.03^{\dagger}$ & 1.71 & 16.96 & $24.18^{\dagger}$ & 1.00 & 0.98 & $0.99^{\dagger}$ \\
\hline Heun's method & 0.59 & $1.09^{\star}$ & $2.04^{\dagger}$ & 1.59 & $4.97^{\star}$ & $22.59^{\dagger}$ & 1.00 & $1.00^{\star}$ & $1.00^{\dagger}$ \\
\hline $2^{\text {nd }}$ order Pade inverse & 0.59 & $1.09^{\star}$ & $2.00^{\dagger}$ & 1.59 & $4.97^{\star}$ & $5.55^{\dagger}$ & 1.00 & $1.00^{\star}$ & $1.00^{\dagger}$ \\
\hline
\end{tabular}

approximant for same observer gain. This is simply because of derivative term dependent prediction coefficient $c_{1}$. Since Euler approximation updates velocity prediction with a higher $c_{1}$ (see Figure 3), it estimates friction better in low gains and is less sensitive to velocity changes, which may occur for high observer gains. It seems that developed adaptive observer and designed Smith predictor based controllers for both step and ramp input tracking exhibit desired performances when both time delay and dynamic friction simultaneously exist.

Remark 5: We note that the Coulomb friction is modeled by discontinuous sign function, which is frequently utilized in sliding mode control. Although this approach theoretically helps one to design control systems with many advantages, its practical implementations may exhibit an undesired behavior called chattering. This behavior is basically an undesired oscillation with finite frequency and amplitude, which is mainly caused by unmodeled dynamics or implementation of control laws in discrete time, see, for example, Lee and Utkin (2007). In Coulomb friction case, this may occur if velocity exhibits oscillations around zero. However, in our simulations with the parameters given in Table 1 we did not encounter chattering. Most possibly this may be due to the fact that in our theoretical framework we did not assume any unmodeled dynamics. Nevertheless, since the numerical simulation is basically a discretization, it may still be possible to encounter chattering due to numerical errors. On the other hand, in any physical implementation, elimination of a possible chattering should be considered carefully. Literature is quite rich 
on this area, and in such a case a suitable chattering suppression technique should be utilized along with the methodology presented here (see e.g. Lee and Utkin (2007) and Bartolini and Punta (2000)). Since our main focus is not on physical implementation, we did not elaborate on this point, which requires and deserves further research.

\section{Conclusion}

In this paper, we considered some control problems related to mechanical systems that contain both friction and measurement delays. We assume that the friction is given by Coulomb model with an unknown but constant coefficient. We proposed an adaptive observer structure to estimate the friction coefficient. We showed that under some reasonable assumptions, which are typical in adaptive control theory, the proposed adaptive structure successfully estimates the friction coefficient with an exponentially decaying error. Hence, by utilizing this estimate, the friction may be canceled by using a simple controller provided that the actual velocity is known. If the velocity measurements are delayed, then a velocity estimator should be employed. To achieve this, we proposed various schemes and considered their effect on the performance of the proposed control structure. Once the effect of friction is eliminated, the resulting system could be considered as a linear time invariant system with a known delay and can be controlled by utilizing classical control techniques. To control the position or velocity separately, we utilized a Smith predictorbased hierarchical control structure. We presented various simulation results which show the performance of the proposed feedback control structure.

The proposed adaptive observer utilizes a general nonlinear function $g(\cdot)$ (see (3)-(4), (32)-(33)). We characterize the class of functions that could be utilized in the observer and for such cases we give some stability results for the proposed adaptive observer structure based on standard Lyapunov stability theory. When the velocity measurements are delayed, a velocity estimation is required. We showed that if the velocity prediction error is small, then the friction parameter estimation is also small as well. This also shows that the proposed structure is robust to such uncertainties.

For velocity estimation, we considered various schemes. As a preliminary research, we have employed first and second order velocity predictors. Consequently, it is inferred that the proposed position tracking system can be fairly utilized as long as the velocity is predicted in a small neighborhood of actual velocities. Especially, when observer gain or dead time is relatively large, friction estimation is hindered as a result of poor velocity prediction. To overcome abrupt changes in states and improve the prediction and estimation performances, reference and derivative filters are utilized. Currently employed first or second order single step predictor designs include derivative blocks that may be very sensitive to velocity changes; therefore, undoubtedly, filtering and predictor designs deserve to further study. As future research, it may be worth investigating different predictor designs such as linear multi step algorithms or higher order filters, which may include inverse dynamics in order to enhance prediction. Also, the selection of an appropriate observer gain to obtain satisfactory estimation performance requires and deserves further study.

When the measurement delay is not known, or is time varying, both velocity prediction and the design of controllers that achieve closed-loop stability become more challenging. This point requires and deserves further research as well. When the friction coefficient is also time varying, it may still be possible to achieve stabilization with the proposed structure under some assumptions due to the robustness of the proposed scheme (see Lemma 3). In fact, simulations reveal that when the actual friction is not confined to Coulomb friction only, the resulting feedback structure may still perform satisfactorily.

\section{Acknowledgements}

The authors would like to thank Prof. Dr. Hitay Özbay for his valuable ideas and support.

\section{Declaration of conflicting interests}

The author(s) declared no potential conflicts of interest with respect to the research, authorship, and/or publication of this article.

\section{Funding}

The author(s) received no financial support for the research, authorship, and/or publication of this article.

\section{ORCID iD}

Caner Odabaş iD https://orcid.org/0000-0002-6890-1003

\section{References}

Armstrong-Hélouvry B, Dupont P and de Wit CC (1994) A survey of models, analysis tools and compensation methods for the control of machines with friction. Automatica 30(7): 1083-1138.

Astrom KJ, Hang CC and Lim B (1994) A new smith predictor for controlling a process with an integrator and long dead-time. IEEE transactions on Automatic Control 39(2): 343-345.

Atkinson KE (1989) An Introduction to Numerical Analysis. 2nd edn. New York: John Wiley.

Bartolini G and Punta E (2000) Chattering elimination with secondorder sliding modes robust to coulomb friction. Journal of Dynamic Systems, Measurement, and Control 122(4): 679-686.

Bliman PA and Sorine M (1993) A system-theoretic approach of systems with hysteresis. Application to friction modelling and compensation. In: Proceedings of the 2nd European Control Conference, Groningen, The Netherlands, 28 June-1 July 1993, pp. 1844-1849. European Union Control Association (EUCA).

Butcher JC and Goodwin N (2008) Numerical Methods for Ordinary Differential Equations, volume 2. Wiley Online Library.

Canudas de Wit C, Olsson H, Åström KJ and Lischinsky P (1995) A new model for control of systems with friction. IEEE Transactions on Automatic Control 40(3): 419-425.

Chapra SC, Canale RP, et al. (2010) Numerical methods for engineers. Boston: McGraw-Hill Higher Education,

Chen Z, Li C, Yao B, Yuan M and Yang C (2020) Integrated coordinated/synchronized contouring control of a dual-linear-motor-driven gantry. IEEE Transactions on Industrial Electronics 67(5): 3944-3954. 
Chen Z, Yao B and Wang Q (2013) Accurate motion control of linear motors with adaptive robust compensation of nonlinear electromagnetic field effect. IEEE/ASME Transactions on Mechatronics 18(3): 1122-1129.

Cicco LD, Mascolo S and Niculescu SI (2011) Robust stability analysis of Smith predictor-based congestion control algorithms for computer networks. Automatica 47(8): 1685-1692.

Dahl PR (1968) A solid friction model. Technical report, Aerospace Corp El Segundo Ca.

Feliu-Batlle V and Rivas-Perez R (2019) Smith predictor based fractional-order integral controller for robust temperature control in a steel slab reheating furnace. Transactions of the Institute of Measurement and Control 41(16): 4521-453.

Francis B and Wonham W (1976) The internal model principle of control theory. Automatica 12(5): 457-465.

Friedland B and Mentzelopoulou S (1992) On adaptive friction compensation without velocity measurement. In: Proceedings of $1 \mathrm{st}$ IEEE Conference on Control Applications, Dayton, OH, USA, 1992, Vol. 2, pp. 1076-1081. IEEE.

Friedland B and Park Y (1992) On adaptive friction compensation. IEEE Transactions on Automatic Control 37(10): 1609-1612.

Khalil HK (2002) Nonlinear Systems. 3rd edn. Upper Saddle River, NJ: Prentice-Hall.

Lee H and Utkin VI (2007) Chattering suppression methods in sliding mode control systems. Annual Reviews in Control 31(2): 179-188.

Lee HS and Tomizuka M (1996) Robust motion controller design for high-accuracy positioning systems. IEEE Transactions on Industrial Electronics 43(1): 48-55.

Lischinsky P, Canudas de Wit C and Morel G (1999) Friction compensation for an industrial hydraulic robot. IEEE Control Systems Magazine 19(1): 25-32.

Majhi S and Atherton DP (2000) Obtaining controller parameters for a new Smith predictor using autotuning. Automatica 36(11): $1651-1658$

Matausek MR and Micic AD (1996) A modified smith predictor for controlling a process with an integrator and long dead-time. IEEE Transactions on Automatic Control 41(8): 1199-1203.

Matausek MR and Micic AD (1999) On the modified smith predictor for controlling a process with an integrator and long dead-time. IEEE Transactions on Automatic Control 44(8): 1603-1606.

Odabaş C (2014) Observer Based Friction Cancellation in Mechanical Systems. Master's Thesis, Bilkent University.

Odabaş C and Morgül (2014) Observer based friction cancellation in mechanical systems. In: 2014 14th International Conference on Control, Automation and Systems (ICCAS 2014), Seoul, South Korea, 22-25 October 2014, pp. 12-16. IEEE.
Olsson H and Astrom KJ (1996) Observer-based friction compensation. In: Proceedings of 35th IEEE Conference on Decision and Control, Kobe, Japan, 13 December 1996, Vol. 4. pp. 4345-4350. IEEE.

Olsson H, Åström K, de Wit CC, et al. (1998) Friction models and friction compensation. European Journal of Control 4(3): 176-195.

Ozbay H (1999) Introduction to Feedback Control Theory. 1st edn. Boca Raton, FL: CRC Press, Inc.

Ray LR, Ramasubramanian A and Townsend J (2001) Adaptive friction compensation using extended Kalman-Bucy filter friction estimation. Control Engineering Practice 9(2): 169-179.

Sastry S and Bodson M (2011) Adaptive Control: Stability, Convergence and Robustness. North Chelmsford, Massachusetts, USA: Courier Corporation.

Smith OJ (1957) Closer control of loops with dead time. Chemical Engineering Progress 53(5): 217-219.

Taşdelen U (2013) On Smith predictor-based controller design for systems with integral action and time delay. Master's Thesis, Bilkent University.

Taşdelen U and Özbay H (2013) On smith predictor-based controller design for systems with integral action and time delay. In: 9th Asian Control Conference (ASCC), Istanbul, 23-26 June 2013, pp. 1-6. IEEE.

Tafazoli S, de Silva CW and Lawrence PD (1998) Tracking control of an electrohydraulic manipulator in the presence of friction. IEEE Transactions Control Systems Technology 6(3): 401-411.

Uyanik İ, Ankarali MM, Cowan NJ, et al. (2015) Independent estimation of input and measurement delays for a hybrid vertical spring-mass-damper via harmonic transfer functions. IFACPapersOnLine 48(12): 298-303.

Vajta M (2000) Some remarks on padé-approximations. In: Proceedings of the 3rd TEMPUS-INTCOM Symposium Veszprém, Hungary, 9-14 September 2000, vol. 242.

Visioli A (2006) Practical PID Control. London: Springer Science \& Business Media.

Wang QG and Zhang Y (2001) Robust identification of continuous systems with dead-time from step responses. Automatica 37(3): 377-390.

Watanabe K and Ito M (1981) A process-model control for linear systems with delay. IEEE Transactions on Automatic Control 26(6): $1261-1269$

Xie W (2007) Sliding-mode-observer-based adaptive control for servo actuator with friction. IEEE Transactions on Industrial Electronics 54(3): 1517-1527.

Youla D, Jabr H and Bongiorno J (1976) Modern wiener-hopf design of optimal controllers-part ii: The multivariable case. IEEE Transactions on Automatic Control AC-21(3): 319-338. 